Research Article

\title{
Optimal Design of Fault-Tolerant Controller for an Electric Power Steering System with Sensor Failures Using Genetic Algorithm
}

\author{
Xue Liu $\mathbb{D}^{1,2}$ Hui Pang $\mathbb{D}^{1,2}$ Yuting Shang $\mathbb{D}^{1,2}$ and Wen $W u^{1}$ \\ ${ }^{1}$ Key Laboratory of Road Construction Technology and Equipment, Chang'an University, Xi'an 710064, China \\ ${ }^{2}$ School of Mechanical and Precision Instrument Engineering, Xi'an University of Technology, Xi'an 710048, China \\ Correspondence should be addressed to Hui Pang; huipang@163.com
}

Received 24 April 2018; Accepted 26 August 2018; Published 9 October 2018

Academic Editor: Rui Moreira

Copyright (C) 2018 Xue Liu et al. This is an open access article distributed under the Creative Commons Attribution License, which permits unrestricted use, distribution, and reproduction in any medium, provided the original work is properly cited.

\begin{abstract}
This paper focuses on the fault-tolerant control (FTC) problem for an electric power steering (EPS) system subjected to stochastic sensor failures, and a novel fault-tolerant controller is proposed based on the genetic algorithm (GA). A mathematical model of the EPS system with sensor failures is first established, and the state feedback control law is solved by using linear quadratic regulator techniques to stabilize the closed-loop control system. Then, the dynamic response errors of the EPS system with and without sensor faults are chosen as the optimization objective function. Furthermore, the appropriate weighting matrices are evaluated to obtain the optimal fault control law by using GA. Finally, simulation results are presented to illustrate the effectiveness of the proposed control strategy.
\end{abstract}

\section{Introduction}

In recent years, electric power steering (EPS) system has been widely equipped in vehicle chassis for improving handling stability and riding comfort, and its control strategy has been proved to be an important approach to enhance vehicle performance [1]. However, the control logics of EPS system are getting much too complicated with increasing requirements and installation of more sensors [2]. Generally, EPS system is subjected to some catastrophic faults such as intermittent sensor connection, complete sensor outage etc., which usually results in severe consequences [3]. For example, complete sensor outage may lead to steering failures and adversely affect vehicle drivability and life span. Therefore, the development of effective control system against sensor failures is a potential research topic from both academic and industrial perspectives.

To this end, a number of researchers have devoted their efforts to conduct various studies in this field on the basis of hardware or analytical redundancy. For instance, Andersson [4] derived an algorithm using three sensors to generate residuals, which serves as a fault detection and isolation module for a quadrotor-unmanned-aerial-vehicle. Isermann et al. [5] also presented several FTC strategies with redundant sensors for the EPS system. Nevertheless, these methods usually lead to a high cost and difficulties in practical application, while the use of analytical redundancy can avoid these limitations.

The analytical fault-tolerant operation can be achieved either passively by employing a control law designed to be insensitive to some known faults, or actively by a fault detection and isolation mechanism, and the redesign of a new control law. For instance, Ghimire et al. [6] developed a fault detection and diagnosis module in an EPS system, but the fault-tolerant control strategy is not considered. To better solve the problem, Lawson and Chen [7] developed an active fault-tolerant controller based on Luenberger observer. If the residual exceeds a threshold with the voltage loss signal, the estimated signal replaces the faulty torque sensor as a command to the steering assist motor. Besides, Cholakkal and Chen [8] developed a robust observer considering the model uncertainties and nonlinear complexities to perform fault-tolerant control strategy. However, a precise and real-time diagnose scheme of statistic failures is still 
a significant challenge in fault-tolerant controller design. Consequently, a number of passive FTC methods are proposed to keep system stability. Wang et al. [9], Tohidi et al. [10], and Zhang et al. [11] designed the robust fault-tolerant controllers against the actuator faults or the sensor faults, whereby the state feedback control laws are obtained by using Lyapunov inequality to keep system stable with faults. But neither of them guaranteed the prescriptive control performance. As for the EPS system, Wang et al. [12] described a fault-tolerant controller based on the linear quadratic regulator (LQR) technique through Riccati equation. The cosimulation and road test are then carried out [13]. Since the selection of LQR weighting matrices for this controller design depended on a trial and error procedure, the global stability-guaranteed FTC design may not be achieved when encountering multisensor faults.

To overcome the limitations of selecting weighting matrices by empirical rules, genetic algorithm (GA) approach is adopted to determine the gain matrix of LQR controller [14-17]. Moreover, to our best knowledge, there only exist few studies on the design of LQR-based faulttolerant controller for the EPS system, which provides the aspiration and motivates this study.

The purpose of this paper is to develop a new faulttolerant controller design based on LQR technique for an EPS system using GA approach, which is useful in promoting the commercialization of various vehicles. To design the controller, an optimization objective function is derived from the dynamic response errors of the EPS system with and without sensor faults. Then, the appropriate weighting matrices $\mathbf{Q}$ and $\mathbf{R}$ are quickly evaluated to obtain the optimal fault control law by using GA. Finally, simulation results validate the effectiveness of the proposed FTC method. The remainder of this paper is organized as follows. The EPS system modeling is formulated in Section 2. Section 3 contains the design of EPS fault-tolerant controller in detail. Simulation comparisons between the normal system and sensor fault system are provided in Section 4, and Section 5 contains the conclusions and future works.

Notations $\mathbf{R}^{n}$ and $\mathbf{R}^{m \times n}$ denote the $n$-dimensional Euclidean space and the space of $m \times n$ real matrices, respectively. $\mathbf{P}>0$ (respectively, $\mathbf{P} \geq 0$ ) means that $\mathbf{P}$ is positive definite (respectively, positive semidefinite). $\mathbf{A}^{\mathrm{T}}$ denotes the transpose of a matrix $\mathbf{A}$. The spectral norm of a matrix $\mathbf{A}$ is denoted as $\|\mathbf{A}\|$.

\section{System Modeling and Problem Formulation}

The model of EPS system is shown in Figure 1. When a car begins to turn, a sensor detects the steering torque $T_{\mathrm{c}}$. Then, the measured torque $T_{\mathrm{c}}$ is used as an approximation to determine the amount of assist torque $T_{\mathrm{a}}$ provided by the electric motor. The amount of assist torque $T_{\mathrm{a}}$ is typically calculated from the tunable torque boost based on the vehicle's speed $v$ and the steering torque $T_{\mathrm{c}}$. Finally, $T_{\mathrm{a}}$ combined with the driver's torque $T_{\mathrm{h}}$ consist of $T_{\mathrm{c}}$. It can be concluded that the EPS system establishes a relationship between the steering mechanism, the motor's electrical dynamics, and the resistant force from the road [18].

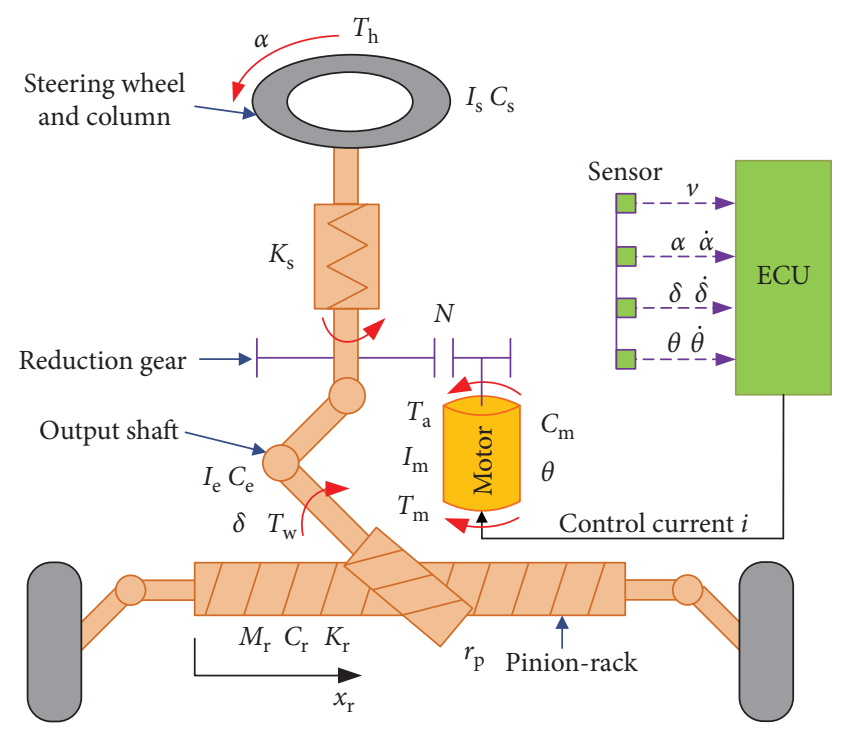

FIgURE 1: Electric power steering model.

Moreover, the key components of EPS system include steering column, reduction gear, output shaft, pinion-rack, motor, and electric control unit (ECU).

According to $[19,20]$, the dynamic equations of the steering column dynamics, output shaft dynamics, and pinion-rack dynamics for the EPS system are described as follows:

$$
\begin{aligned}
& I_{\mathrm{s}} \ddot{\alpha}+C_{\mathrm{s}} \dot{\alpha}=T_{\mathrm{h}}-K_{\mathrm{s}}(\alpha-\delta), \\
& I_{\mathrm{e}} \ddot{\delta}+C_{\mathrm{e}} \dot{\delta}=K_{\mathrm{s}}(\alpha-\delta)+N T_{\mathrm{a}}-T_{\mathrm{w}}, \\
& M_{\mathrm{r}} \ddot{x}_{\mathrm{r}}+C_{\mathrm{r}} \dot{x}_{\mathrm{r}}+K_{\mathrm{r}} x_{\mathrm{r}}=\frac{T_{\mathrm{w}}}{r_{\mathrm{p}}-F_{\mathrm{R}}} .
\end{aligned}
$$

Typically, the motor used in EPS is a DC motor, and the dynamics of the motor are given by

$$
\begin{aligned}
I_{\mathrm{m}} \ddot{\theta}+C_{\mathrm{m}} \dot{\theta} & =T_{\mathrm{m}}-T_{\mathrm{a}}, \\
T_{\mathrm{m}} & =\frac{K_{\mathrm{a}}}{R}\left(u-K_{\mathrm{b}} \dot{\theta}\right) .
\end{aligned}
$$

The motor shaft is mechanically coupled with the pinion shaft via a reduction gear with ration $N$, which eventually results in the assist torque as

$$
T_{\mathrm{a}}=K_{\mathrm{m}}(\theta-N \delta) .
$$

Also, the resistance term can be concluded as [21]

$$
F_{\mathrm{TR}}=K_{\mathrm{r}} x_{\mathrm{r}}+F_{\mathrm{R}} .
$$

Note that the resistance force on the rack is mainly $K_{\mathrm{r}} x_{\mathrm{r}}$, and $F_{\mathrm{R}}$ is the road disturbances.

Depending on the angular deformation, $T_{\mathrm{c}}$ acted on the steering column can be detected by sensor as

$$
T_{\mathrm{c}}=K_{\mathrm{s}}(\alpha-\delta) .
$$

The pinion and output shaft are connected by constant velocity joints, thus $x_{\mathrm{r}}=\delta \cdot r_{\mathrm{p}}$. 
Correspondingly, the state-space equation of the EPS system is expressed as

$$
\left\{\begin{array}{l}
\dot{\mathbf{X}}=\mathbf{A X}+\mathbf{B U} \\
\mathbf{Y}=\mathbf{C X}+\mathbf{D U}
\end{array}\right.
$$

where $\mathbf{X}=\left[\begin{array}{llllll}\alpha & \delta & \theta & \dot{\alpha} & \dot{\delta} & \theta\end{array}\right]^{\mathrm{T}}$ and $\mathbf{U}=\left[\begin{array}{lll}T_{\mathrm{h}} & F_{\mathrm{R}} & u\end{array}\right]^{\mathrm{T}}$. Note that the velocity signal $v$ is linear with the motor voltage $u$ via a tunable boost curve, and the assist motor torque and the column torque are considered as the outputs of the system such that $\mathbf{Y}=\left[\begin{array}{ll}T_{\mathrm{a}} & T_{\mathrm{c}}\end{array}\right]^{\mathrm{T}} . \mathbf{A}, \mathbf{B}, \mathbf{C}$, and $\mathbf{D}$ are the known matrices with appropriate dimensions (Appendix).

It is observed from (7) that the pair (A, B) is completely controllable and the pair $(\mathbf{A}, \mathbf{C})$ is observable. A state feedback control scheme can be employed, and the full-state feedback controller can be defined as

$$
\mathbf{U}=\mathbf{r}-\mathbf{K X},
$$

where $\mathbf{r}$ is the reference input. Since the complete sensor outage is considered as the worst case in the EPS system, the sensor faults function matrices are described as $\mathbf{M}=\operatorname{diag}\left\{f_{1}, f_{2}, \ldots, f_{6}\right\}$, wherein $\mathbf{M}$ is a $6 \times 6$ identity matrix if all sensors are in good working condition. A failure in the $i^{\text {th }}$ sensor is modeled by setting the $i^{\text {th }}$ diagonal element in $\mathbf{M}$ to zero, that is, $f_{i}=0$. By denoting $\mathbf{U}_{f}$ as the controller with possible sensor failure, we have

$$
\mathbf{U}_{f}=\mathbf{r}-\mathbf{K M X} \text {. }
$$

By further derivation, the system with possible sensor failures can be given by

$$
\left\{\begin{array}{l}
\dot{\mathbf{X}}_{f}=(\mathbf{A}-\mathbf{B K M}) \mathbf{X}_{f}+\mathbf{B r}, \\
\mathbf{Y}_{f}=\mathbf{C X}_{f},
\end{array}\right.
$$

where $\mathbf{X}_{f}$ is the state vector and $\mathbf{Y}_{f}$ is the output vector when the sensor failures occur. To fulfill the complete faulttolerant control for (10), we need to find out an optimal gain matrix $\mathbf{K}$ such that the closed-loop system (10) with controller (9) can keep stability with possible sensor failures.

\section{Optimal Design of Fault-Tolerant Controller}

3.1. EPS Fault-Tolerant Controller Design. In this section, a fault-tolerant controller is designed to make the EPS system robust against sensor failures. To design this controller, the following linear quadratic performance index for (10) is defined based on LQR technique [22]:

$$
J_{\infty}=\int_{0}^{\infty} e^{2 \lambda t}\left(\mathbf{X}_{f}^{T} \mathbf{Q} \mathbf{X}_{f}+\mathbf{U}_{f}^{T} \mathbf{R} \mathbf{U}_{f}\right) d t,
$$

where the attenuation $\lambda>0$ and $\mathbf{U}_{f}$ is the control input vector, respectively, and $\mathbf{Q} \geq 0$ and $\mathbf{R}>0$ are parameter matrices to be designed. The performance index $J_{\infty}$ weighted by $\mathbf{Q}$ and $\mathbf{R}$ establishes a trade-off between the control speed and the energy used. The objective for this controller design is to find out an appropriate control input $\mathbf{U}_{f}$ with sensors being ineffective and minimize $J_{\infty}$. To fulfill the controller design, thus the following lemma and theorems are introduced.
Lemma 1 [23]. The linear autonomous system is exponentially stable if and only if there exists $\mathbf{X}=\mathbf{X}^{\mathrm{T}}$ such that $\mathbf{X}>0$ and $\mathbf{A}^{\mathrm{T}} \mathbf{X}+\mathbf{X} \mathbf{A}<0$.

Theorem 1. Consider the closed-loop system given in (10); $\mathbf{Q}$ and $\mathbf{R}$ are the weighting matrices used in the linear quadratic performance index. If $\mathbf{Q}, \mathbf{R}$, and the positive define matrix $\mathbf{P}$ satisfy

$$
\left(\mathbf{M}_{i}-\mathbf{I}_{n}\right) \mathbf{P B R}{ }^{-1} \mathbf{B}^{\mathrm{T}} \mathbf{P}+\mathbf{P B R}^{-1} \mathbf{B}^{\mathrm{T}} \mathbf{P} \mathbf{M}_{i}+\mathbf{Q}>0 .
$$

The gain matrix $\mathbf{K}=\mathbf{R}^{-1} \mathbf{B}^{\mathrm{T}} \mathbf{P}$ for the fault-tolerant controller in (9) can guarantee the stability of the system with possible sensor failure, which means

$$
\operatorname{Re}\left\{\lambda\left(\mathbf{A}-\mathbf{B K M}_{i}\right)\right\}<-\alpha .
$$

Proof. Based on [24, 25], the performance index of (11) is minimized with

$$
\mathbf{K}=\mathbf{R}^{-1} \mathbf{B}^{\mathrm{T}} \mathbf{P},
$$

where $\mathbf{P}$ is the solution of the following algebraic Riccati equation:

$$
\left(\mathbf{A}+\alpha \mathbf{I}_{n}\right)^{\mathrm{T}} \mathbf{P}+\mathbf{P}\left(\mathbf{A}+\alpha \mathbf{I}_{n}\right)-\mathbf{P B R}^{-1} \mathbf{B}^{\mathrm{T}} \mathbf{P}+\mathbf{Q}=0 .
$$

Substituting (14) in (13) yields

$$
\operatorname{Re}\left\{\lambda\left(\mathbf{A}+\alpha \mathbf{I}_{n}-\mathbf{B R}^{-1} \mathbf{B}^{\mathrm{T}} \mathbf{P} \mathbf{M}_{i}\right)\right\}<0 .
$$

According to Lemma 1, (16) is equivalent to

$$
\left(\mathbf{A}+\alpha \mathbf{I}_{n}-\mathbf{B R}^{-1} \mathbf{B}^{\mathrm{T}} \mathbf{P} \mathbf{M}_{i}\right)^{\mathrm{T}} \mathbf{P}+\mathbf{P}\left(\mathbf{A}+\alpha \mathbf{I}_{n}-\mathbf{B R}^{-1} \mathbf{B}^{\mathrm{T}} \mathbf{P} \mathbf{M}_{i}\right)<0 .
$$

Inequality (17) can be written as

$$
\begin{aligned}
& \left(\mathbf{A}+\alpha \mathbf{I}_{n}\right)^{\mathrm{T}} \mathbf{P}+\mathbf{P}\left(\mathbf{A}+\alpha \mathbf{I}_{n}\right) \\
& \quad-\left(\mathbf{P B R}^{-1} \mathbf{B}^{\mathrm{T}} \mathbf{P} \mathbf{M}_{i}+\mathbf{M}_{i} \mathbf{P B R}^{-1} \mathbf{B}^{\mathrm{T}} \mathbf{P}\right)<0 .
\end{aligned}
$$

Similarly, Equation (15) can be rewritten as

$$
\left(\mathbf{A}+\alpha \mathbf{I}_{n}\right)^{\mathrm{T}} \mathbf{P}+\mathbf{P}\left(\mathbf{A}+\alpha \mathbf{I}_{n}\right)=\mathbf{P B R}^{-1} \mathbf{B}^{\mathrm{T}} \mathbf{P}-\mathbf{Q} .
$$

Substituting (19) in (18), we can easily obtain (12), which completes the proof.

According to Theorem 1, it is possible for the EPS system to have fault-tolerant features with a set of known faults or failures by choosing appropriate $\mathbf{Q}, \mathbf{R}$. Normally, the designer performs searching of $\mathbf{Q}$ and $\mathbf{R}$ by trial and error, which is oriented by experience and easier found to be a local optimal solution. Due to the advantages in searching optimal design parameters and obtaining globally optimal solution, the GA is adopted to find the weighting matrix.

3.2. GA-Based Optimal Design of EPS Fault-Tolerant Controller. To evaluate the optimal $\mathbf{Q}$ and $\mathbf{R}$ and then ultimately obtain the optimal control gain for the EPS 
fault-tolerant controller, we use the GA approach to minimize $J_{\infty}$. The flowchart of GA is shown in Figure 2.

In Figure 2, the key point of GA is to seek an optimized objective function that ranks the performance of each individual by calculating the fitness value. Here, dynamic response errors of EPS system with and without sensor failures are adopted as the optimized function through Lyapunov equation.

Considering the normal system and fault system, a composite system is formulated as

$$
\left\{\begin{array}{l}
\dot{\overline{\mathbf{X}}}=\overline{\mathbf{A X}}+\overline{\mathbf{B}} \mathbf{r} \\
\overline{\mathbf{Z}}=\overline{\mathbf{C X}}
\end{array}\right.
$$

where $\overline{\mathbf{X}}=\left[\begin{array}{ll}\mathbf{X}^{\mathrm{T}} & \mathbf{X}_{f}^{\mathrm{T}}\end{array}\right]^{\mathrm{T}} \in \mathbf{R}^{2 n}$ is the state vector and $\overline{\mathbf{Z}}=\mathbf{Y}-\mathbf{Y}_{f}$ is the output vector considering the output errors of EPS system with and without sensor failures, we have

$$
\begin{aligned}
& \overline{\mathbf{A}}=\left[\begin{array}{cc}
\mathbf{A} & 0 \\
0 & \mathbf{A}-\mathbf{B K M}
\end{array}\right], \\
& \overline{\mathbf{B}}=\left[\begin{array}{l}
\mathbf{B} \\
\mathbf{B}
\end{array}\right] \text {, } \\
& \overline{\mathbf{C}}=\left[\begin{array}{ll}
\mathrm{C} & -\mathrm{C}
\end{array}\right] \text {. }
\end{aligned}
$$

It is assumed that the initial state of the EPS system is zero with the step input, and the optimized function is defined as

$$
\mathbf{J}=\int_{0}^{\infty} \overline{\mathbf{Z}}^{2}(t) d t=\int_{0}^{\infty}\left[\mathbf{Y}(t)-\mathbf{Y}_{\mathbf{f}}(t)\right]^{2} d t
$$

Theorem 2. If there exists appropriate weighting matrices $\mathbf{Q}$, $\mathbf{R}$ and control law $\mathbf{K}$ such that the Lyapunov Equation (23) holds with the positive definite solution $\mathbf{P}$

$$
\overline{\mathbf{A}}^{\mathrm{T}} \mathbf{P}+\mathbf{P} \overline{\mathbf{A}}+\mathbf{Q}_{1}=0
$$

where

$$
\mathbf{Q}_{1}=\left[\begin{array}{cc}
\mathbf{C}^{\mathrm{T}} \mathbf{C} & -\mathbf{C}^{\mathrm{T}} \mathbf{C} \\
-\mathbf{C}^{\mathrm{T}} \mathbf{C} & \mathbf{C}^{\mathrm{T}} \mathbf{C}
\end{array}\right],
$$

with satisfying the following equation:

$$
\overline{\mathbf{C A}}^{-1} \overline{\mathbf{B}}=0 .
$$

When the optimized function $\|\mathbf{J}\|$ reaches its minimum value,

$$
\mathbf{J}=-\overline{\mathbf{B}}^{\mathrm{T}}\left(\overline{\mathbf{A}}^{\mathrm{T}}\right)^{-1} \mathbf{P} \overline{\mathbf{A}}^{-1} \overline{\mathbf{B}}-2 \overline{\mathbf{B}}^{\mathrm{T}} \mathbf{P} \overline{\mathbf{A}}^{-2} \overline{\mathbf{B}}
$$

The control law defined by (14) can guarantee the asymptotic stability of the fault EPS system with a minimum output error.

Proof. Equation (22) can be written as

$$
\mathbf{J}=\int_{0}^{t} \overline{\mathbf{Z}}^{2}(\tau) d \tau
$$

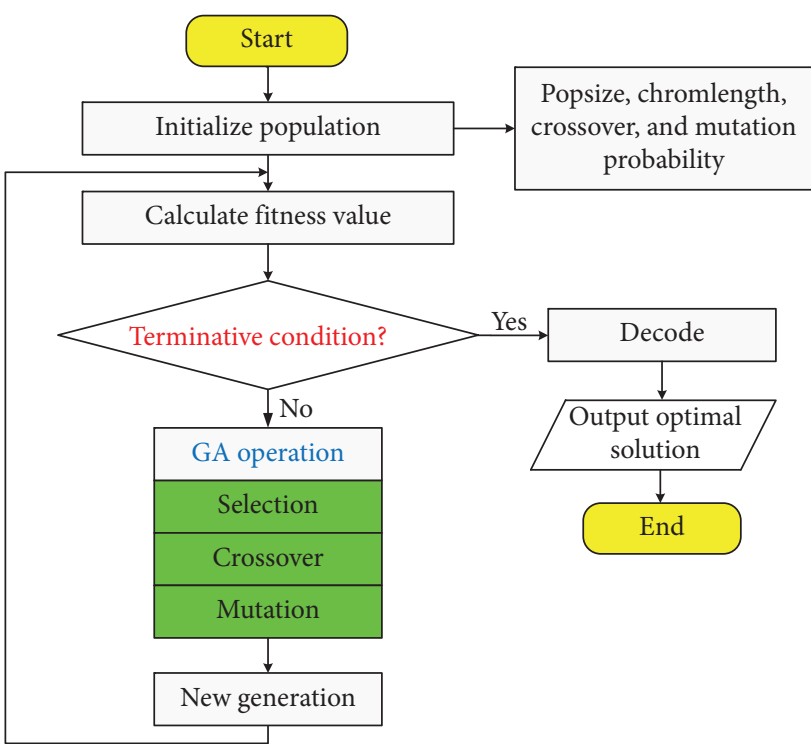

Figure 2: The flowchart of genetic algorithm.

Define the Lyapunov function

$$
V(t)=[\overline{\mathbf{X}}(t)]^{\mathrm{T}} \mathbf{P} \overline{\mathbf{X}}(t),
$$

where $\mathbf{P}$ is the positive definite solution of the Riccati Equation (15).

Substituting (23) and (24) into (27) yields

$$
\mathbf{J}=\int_{0}^{t}[\overline{\mathbf{X}}(\tau)]^{\mathrm{T}} \mathbf{Q}_{1} \overline{\mathbf{X}}(\tau) d \tau .
$$

Deriving along (28) and using (20) gives

$$
\dot{V}(t)=-[\overline{\mathbf{X}}(t)]^{\mathrm{T}} \mathbf{Q}_{1} \overline{\mathbf{X}}(t)+2 \overline{\mathbf{B}}^{\mathrm{T}} \mathbf{P} \overline{\mathbf{X}}(t) .
$$

Integrating Equation (30), we have

$$
V(t)=-J(t)+2 \overline{\mathbf{B}}^{\mathrm{T}} \mathbf{P} \int_{0}^{t} \overline{\mathbf{X}}(\tau) d \tau .
$$

Since the step response with zero-state unit is

$$
\overline{\mathbf{X}}(t)=\int_{0}^{t} e^{\overline{\mathbf{A}} \tau} \overline{\mathbf{B}} d \tau=\overline{\mathbf{A}}^{-1}\left(e^{\overline{\mathbf{A}} t}-\mathbf{I}_{2 n}\right) \overline{\mathbf{B}}
$$

Thus, we have

$$
\int_{0}^{t} \overline{\mathbf{X}}(\tau) d \tau=\overline{\mathbf{A}}^{-2} e^{\overline{\mathbf{A}} t} \overline{\mathbf{B}}-\overline{\mathbf{A}}^{-2} \overline{\mathbf{B}}-\overline{\mathbf{A}}^{-1} \overline{\mathbf{B}} t .
$$

Substituting (33) and (29) into (31) results in

$$
\begin{aligned}
\mathbf{J}= & -\overline{\mathbf{B}}^{\mathrm{T}}\left(\overline{\mathbf{A}}^{\mathrm{T}}\right)^{-1} \mathbf{P} \overline{\mathbf{A}}^{-1} \overline{\mathbf{B}}-2 \overline{\mathbf{B}}^{\mathrm{T}} \mathbf{P} \overline{\mathbf{A}}^{-2} \overline{\mathbf{B}}-\mathbf{H} t+\mathbf{G}, \\
\mathbf{H}= & \overline{\mathbf{B}}^{\mathrm{T}}\left[\left(\overline{\mathbf{A}}^{\mathrm{T}}\right)^{-1} \mathbf{P}+\mathbf{P} \overline{\mathbf{A}}^{-1}\right] \overline{\mathbf{B}}, \\
\mathbf{G}(t)= & 2 \overline{\mathbf{B}}^{\mathrm{T}} \mathbf{P} \overline{\mathbf{A}}^{-2} e^{\overline{\mathbf{A}} t} \overline{\mathbf{B}}+2 \overline{\mathbf{B}}^{\mathrm{T}} e^{\overline{\mathbf{A}} t}\left(\overline{\mathbf{A}}^{\mathrm{T}}\right)^{-1} \\
& \cdot \mathbf{P} \overline{\mathbf{A}}^{-1} \overline{\mathbf{B}}-\overline{\mathbf{B}}^{\mathrm{T}} e^{\overline{\mathbf{A}} t}\left(\overline{\mathbf{A}}^{\mathrm{T}}\right)^{-1} \mathbf{P} \overline{\mathbf{A}}^{-1} e^{\overline{\mathbf{A}} t} \overline{\mathbf{B}} .
\end{aligned}
$$


According to (25), we have

$$
\overline{\mathbf{B}}^{\mathrm{T}}\left(\overline{\mathbf{A}}^{\mathrm{T}}\right)^{-1} \overline{\mathbf{C}}^{\mathrm{T}} \overline{\mathbf{C A}}^{-1} \overline{\mathbf{B}}=0 .
$$

Equation (35) can be written as

$$
\overline{\mathbf{B}}^{\mathrm{T}}\left(\overline{\mathbf{A}}^{\mathrm{T}}\right)^{-1}\left[\overline{\mathbf{A}}^{\mathrm{T}} \mathbf{P}+\mathbf{P} \overline{\mathbf{A}}\right] \overline{\mathbf{A}}^{-1} \overline{\mathbf{B}}=0 .
$$

It is equivalent to

$$
\overline{\mathbf{B}}^{\mathrm{T}}\left[\left(\overline{\mathbf{A}}^{\mathrm{T}}\right)^{-1} \mathbf{P}+\mathbf{P} \overline{\mathbf{A}}^{-1}\right] \overline{\mathbf{B}}=0 .
$$

Thus, we get $\mathbf{H}=0$ in terms of (34).

It is obvious that the EPS system is stable if the following equation holds

$$
\lim _{t \longrightarrow \infty} e^{\overline{\mathbf{A}} t}=0
$$

That means $\mathbf{G}(\infty)=0$, and the proof is completed according to (34), (37), and (38).

According to Theorem 1, Theorem 2, and GA algorithm in Figure 2, the main processes of GA-based weighting matrix selection for the controller design are described as follows:

Step 1. Initialize population. The population size affects both the ultimate performance and the efficiency of GAs. A large population is more likely to contain representatives from a large number of hyperplanes [26]. Hence the GA can perform a more informed search. On the other hand, a large population requires more evaluations per generation, possibly resulting in an unacceptably slow rate of convergence. In this paper, the $\mathbf{Q}$ and $\mathbf{R}$ matrices are assumed to be diagonal, such that $\mathbf{Q}=m \mathbf{I}_{n}$ and $\mathbf{R}=t \mathbf{I}_{n}$, and the ranges are taken as $0<m<50,0<t<2000$, which are based on our previous work and research [27]. The coefficients $m$ and $t$ are set as individual $[m \cdot t]$ to be optimized. In the current experiment, the population size is set to be 50 .

Step 2. Calculate fitness value. For each individual, the $\mathbf{Q}$ and $\mathbf{R}$ matrices are applied in Theorem 2 to obtain the performance index $\|\mathbf{J}\|$ as the fitness value.

Step 3. Terminate condition. The maximum generation is set as 150 . The algorithm is over when the GA exceeds the maximum number of generations and satisfies the constraints in Theorem 1 and Theorem 2. If not, it goes on for the GA operation until it meets the requirements.

Step 4. GA operation. The GA operation includes the selection, crossover, and mutation. The fitness-weight roulette game, which means that the fittest individuals have a greater chance of survival than weaker ones, is chosen as the selection strategy. The crossover rate controls the frequency with which the crossover operator is applied. The higher the crossover rate, the more quickly new structures are introduced into the population. Mutation is a secondary search operator which increases the variability of the population. A low level of mutation serves to prevent any given bit position from remaining converged to a single value in the entire population. A high level of mutation rate causes an essentially random search. In this paper, the crossover rate and the mutation rate are set as $0.8,0.1$, respectively. In every generation, the fittest individuals are randomly selected and are recombined and randomly mutated to form a new generation which goes to the step 2 .

In order to improve the optimal weight coefficients of the $\mathbf{Q}$ and $\mathbf{R}$ matrices, the corresponding weight coefficients are calculated as $m=4.54, t=10.22$ by GA when the iteration of generation arrives 150 with the constraint satisfied. Thus, the matrix $\mathbf{K}$ is obtained.

Note that the proposed control design method can ensure the global sensor fault system's dynamic output to be as close as the normal system's output. The block diagram of the GAbased EPS fault-tolerant controller is shown in Figure 3.

Remark 1. The system matrix of reference normal system can be changed as $\mathbf{A}-\mathbf{B K}_{0} . \mathbf{K}_{0}$ is derived from LQR technique to minimize the performance index $J_{\infty}$, which can optimize the performance of the normal EPS system.

\section{Simulation and Analysis}

In order to verify the effectiveness and applicability of the proposed fault-tolerant controller, a numerical case is investigated in this section. The EPS system's parameters and variables used for the simulation are shown in Table 1.

According to the above parameters, by evaluating the GA-based EPS fault-tolerant controller, the gain matrix $\mathbf{K}$ is obtained as follows:

$$
\mathbf{K}=\left[\begin{array}{cccccc}
-6.2843 & 68.4608 & -2.27 e-05 & 0.1152 & 0.0526 & -3.17 e-08 \\
0.2095 & -1.0061 & -1.95 e-08 & -4.23 e-04 & -0.0014 & 1.91 e-10 \\
-8.69 e-05 & -7.7 e-05 & 0.1390 & -5.29 e-08 & -3.94 e-08 & 0.5842
\end{array}\right]
$$

The step and bump responses of $T_{\mathrm{h}}$ to $T_{\mathrm{a}}$ with one sensor failure are shown in Figures 4 and 5, respectively. It is noted that the comparisons of $T_{\mathrm{a}}$ for the normal EPS system and the fault one with every sensor failure are provided in both of these two figures.
It can be seen from Figures 4 and 5 that there is a smaller persistent deviation for the output response of the EPS system in the presence of failure in sensor 1 , sensor 3 , or sensor 5, respectively, compared with the normal EPS system. Note that the fault EPS system can reach stability after 


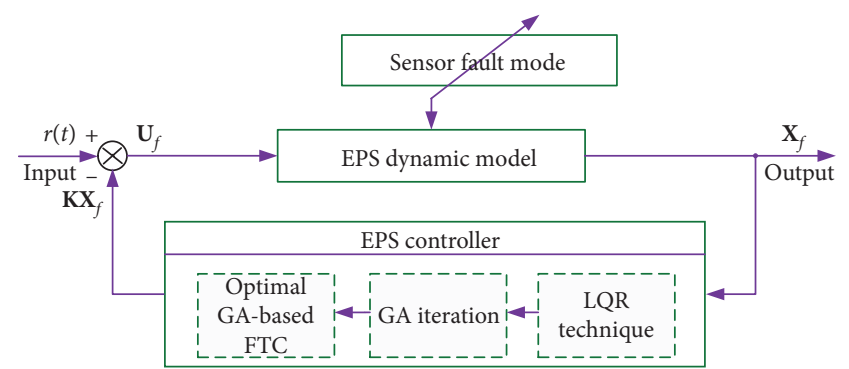

Figure 3: The design of fault-tolerant controller.

TABLE 1: The parameters of EPS.

\begin{tabular}{lc}
\hline Parameter & Value \\
\hline$I_{\mathrm{s}}\left(\mathrm{kg} \cdot \mathrm{m}^{2}\right)$ & 0.0012 \\
$C_{\mathrm{s}}(\mathrm{N}-\mathrm{m}-\mathrm{s} / \mathrm{rad})$ & 0.261 \\
$K_{\mathrm{s}}(\mathrm{N}-\mathrm{m} / \mathrm{rad})$ & 184.33 \\
$I_{\mathrm{m}}\left(\mathrm{kg}-\mathrm{m}^{2}\right)$ & 0.00018 \\
$C_{\mathrm{m}}(\mathrm{N}-\mathrm{m}-\mathrm{s} / \mathrm{rad})$ & 0.00339 \\
$M_{\mathrm{r}}(\mathrm{kg})$ & 0.89 \\
$K_{\mathrm{r}}(\mathrm{N} / \mathrm{m})$ & 91061.4 \\
$N(-)$ & 16.5 \\
$I_{\mathrm{e}}\left(\mathrm{kg}-\mathrm{m}^{2}\right)$ & 0.001 \\
$C_{\mathrm{e}}(\mathrm{N}-\mathrm{m}-\mathrm{s} / \mathrm{rad})$ & 0.031 \\
$r_{\mathrm{p}}(\mathrm{m})$ & 0.007 \\
$R(\Omega)$ & 0.67 \\
$K_{\mathrm{m}}(\mathrm{N}-\mathrm{m} / \mathrm{rad})$ & 125 \\
$K_{\mathrm{a}}(\mathrm{N}-\mathrm{m} / \mathrm{A})$ & 0.04 \\
$K_{\mathrm{b}}\left(\mathrm{V} / \mathrm{rad}-\mathrm{s}^{-1}\right)$ & 0.057 \\
\hline
\end{tabular}

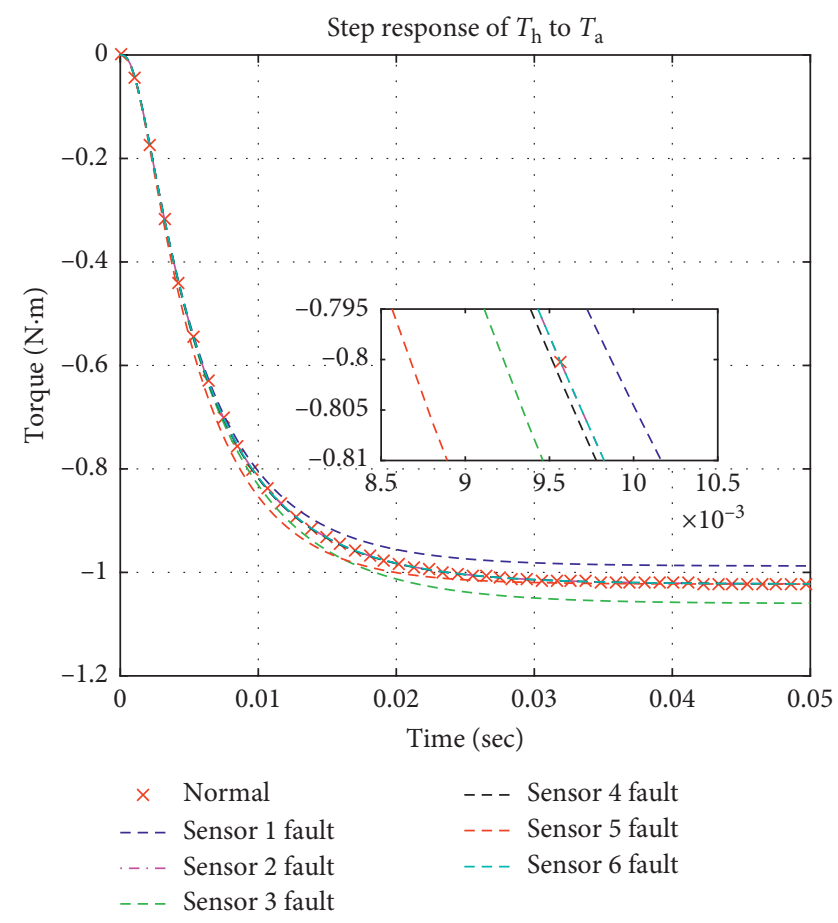

FIGURE 4: The step response of EPS system with a sensor failure.

0.03 seconds and the output response is still in an acceptable range when occurring sensor fault. Besides, the output

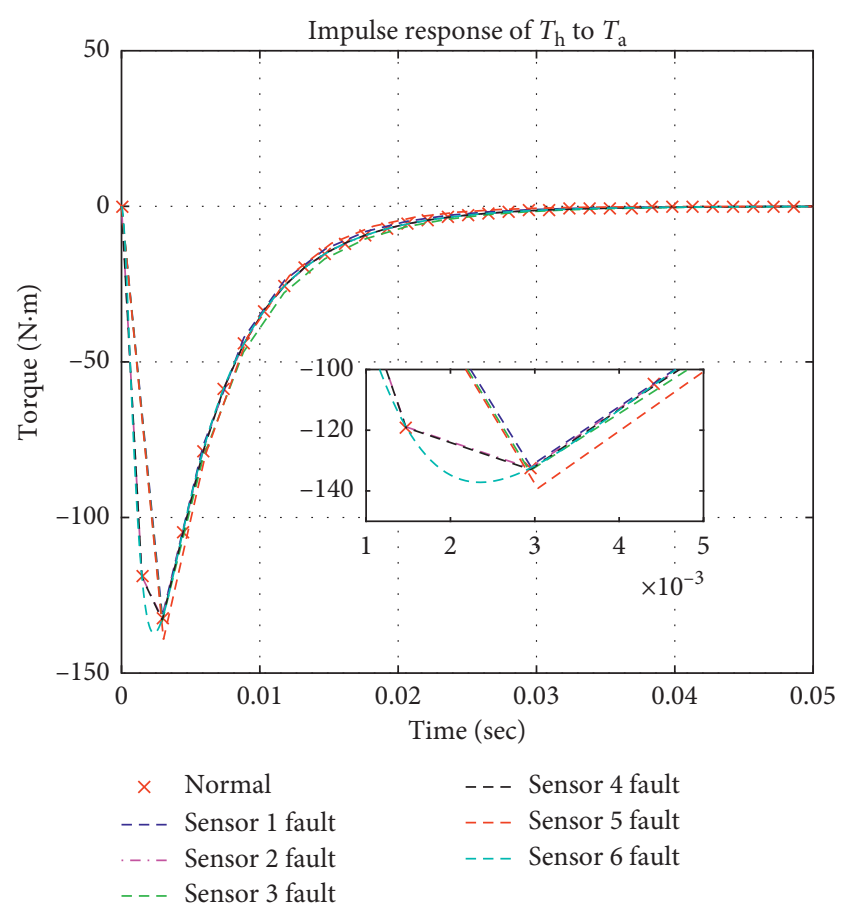

FIgURE 5: The bump response of EPS system with a sensor failure.

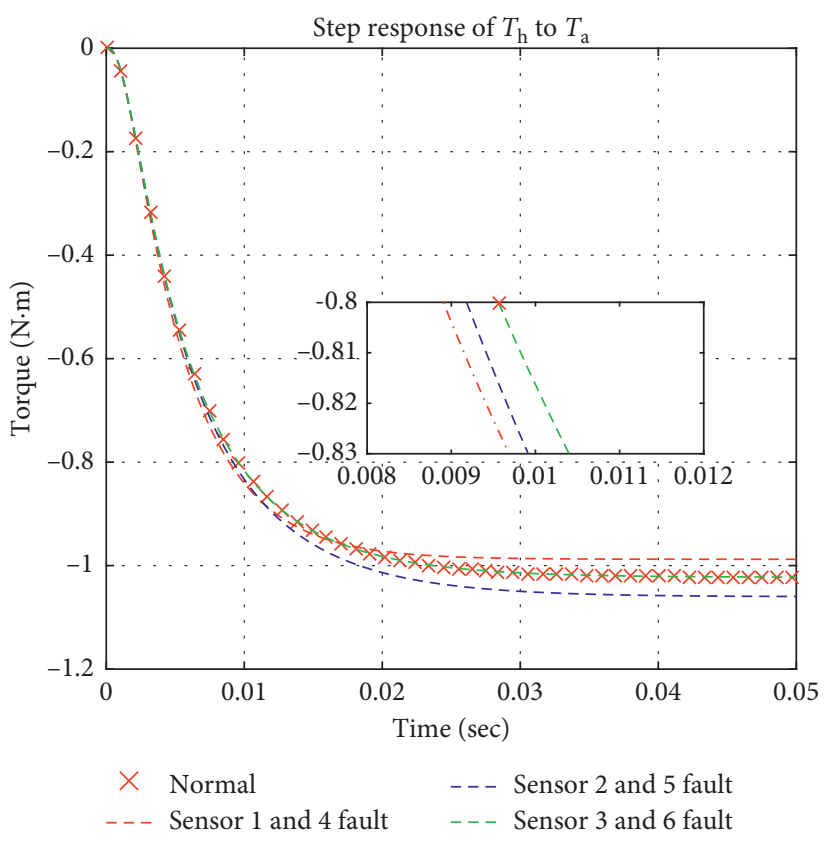

FIgURE 6: The step response of EPS system with two sensor failure.

responses of the normal and fault EPS system are nearly identical to each other in the presence of failure in sensor 2, sensor 4, or sensor 6, respectively, which illustrates the proposed fault-tolerant controller has some positive effects on the fault EPS system.

To verify the effectiveness of this controller, the step and bump responses of $T_{\mathrm{h}}$ to $T_{\mathrm{a}}$ with two sensors failures at the same time are shown in Figures 6 and 7, respectively. It is observed from Figure 6 that the output performance of the 


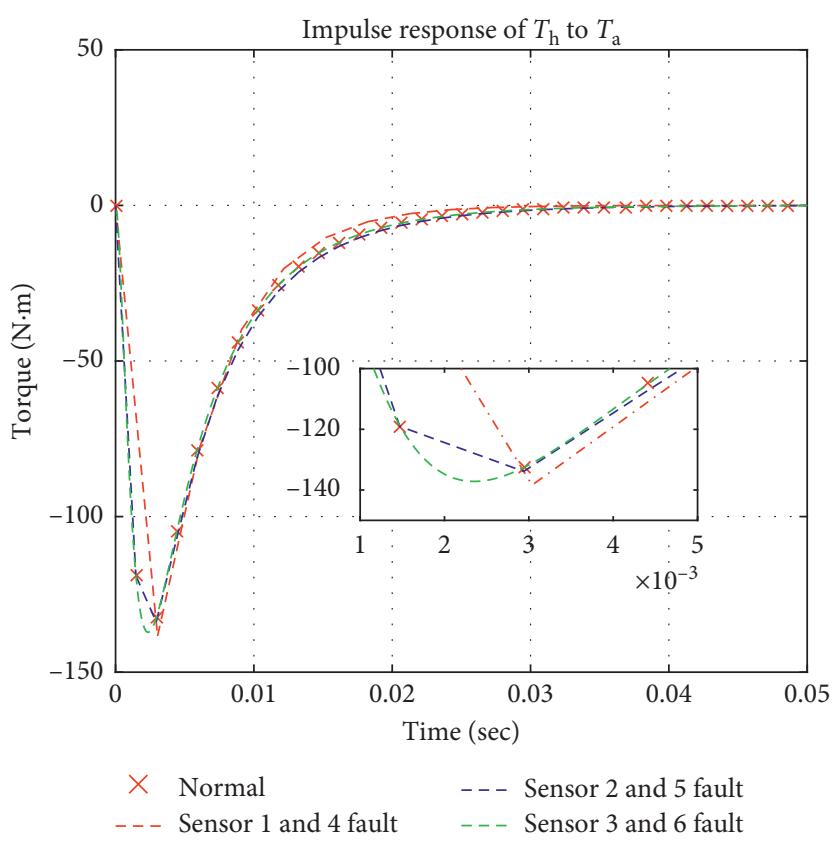

Figure 7: The impulse response of EPS system with two sensor failure.

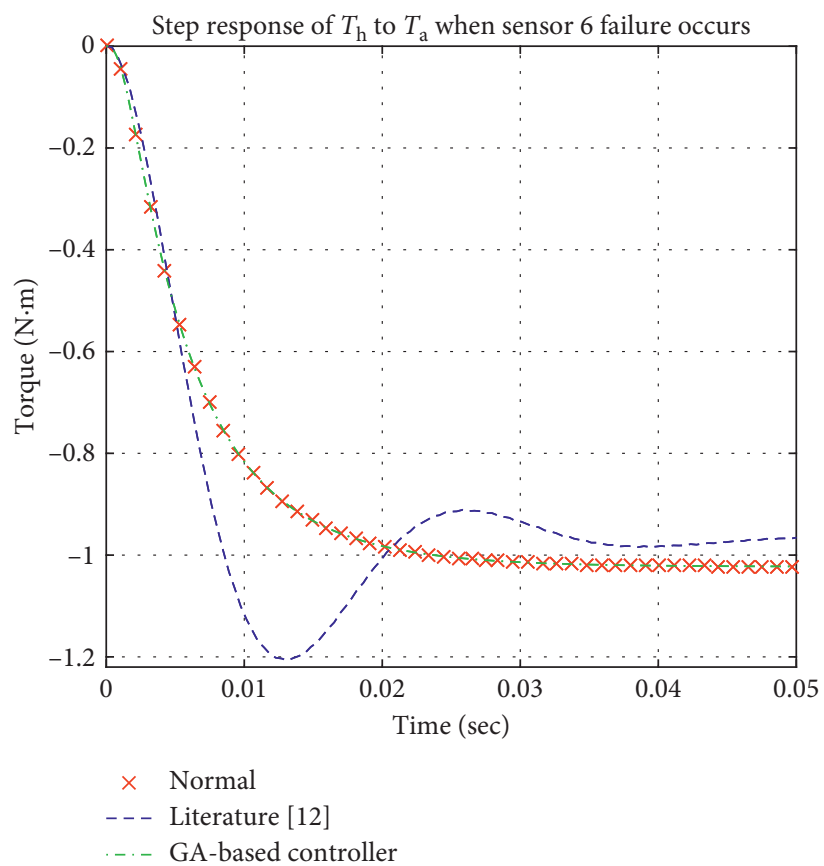

Figure 8: The step response of EPS system with the failure of sensor 6.

EPS system is deteriorated with the failures of sensor 1 and 4 , sensor 2 and 5, and sensor 3 and 6, respectively, while it is still in an acceptable stability region compared with the normal EPS system. In Figure 7, the EPS system with two sensor failures can reach stability within $0.04 \mathrm{~s}$ under a sudden bump road disturbance, which validates the reliability and effectiveness of the proposed optimal faulttolerant controller.
To examine the advantages over the existing control method for the EPS system, Figure 8 shows the comparison between the step response of $T_{\mathrm{h}}$ to $T_{\mathrm{a}}$ by using the control scheme in the literature [12] and our proposed GA-based fault-tolerant controller with one sensor failure, typically sensor 6 . Note that the output performance of the normal EPS system is also included. It is obvious that the method in [12] has oscillations in time domain, while the proposed 
GA-based fault-tolerant controller works well and can closely track with the normal system.

\section{Conclusion}

In this article, an optimal design method of fault-tolerant controller was proposed for an electric power steering system with possible sensor failures based on GA approach. LQR technique is used to construct fault-tolerant control law for the system. Then, the appropriate weighting matrices $\mathbf{Q}$ and $\mathbf{R}$ used in LQR technique are evaluated to obtain the optimal fault control law by using GA, which guarantee the output of sensor fault system closely tracking the normal reference system. Furthermore, the simulation shows that the proposed method can obtain better disturbance attenuation performance when encountering with different sensor fault mode. Since the external disturbances and model uncertainty are inevitable in practical EPS system, future work will focus on investigating the robustness of the GA-based LQR control scheme.

\section{Appendix}

The coefficient matrices of A, B, C, D for Equation (7) are expressed as [28]

$$
\begin{aligned}
& \mathbf{A}=\left[\begin{array}{cccccc}
0 & 0 & 0 & 1 & 0 & 0 \\
0 & 0 & 0 & 0 & 1 & 0 \\
0 & 0 & 0 & 0 & 0 & 1 \\
-\frac{K_{\mathrm{s}}}{I_{\mathrm{s}}} & \frac{K_{\mathrm{s}}}{I_{\mathrm{s}}} & 0 & -\frac{C_{\mathrm{s}}}{I_{\mathrm{s}}} & 0 & 0 \\
\frac{K_{\mathrm{s}}}{M_{\mathrm{R}} r_{\mathrm{p}}^{2}}-\frac{1}{M_{\mathrm{R}} r_{\mathrm{p}}}\left(K_{\mathrm{r}} r_{\mathrm{p}}+\frac{K_{\mathrm{s}}}{r_{\mathrm{p}}}+N^{2} K_{\mathrm{m}} / r_{\mathrm{p}}\right) & \frac{N K_{\mathrm{m}}}{M_{\mathrm{R}} r_{\mathrm{p}}^{2}} & 0 & -\frac{C_{\mathrm{R}}}{M_{\mathrm{R}}} & 0 \\
0 & \frac{K_{\mathrm{m}} N}{I_{\mathrm{m}}} & -\frac{K_{\mathrm{m}}}{I_{\mathrm{m}}} & 0 & 0 & -\frac{C_{\mathrm{m}} R+K_{\mathrm{a}} K_{\mathrm{b}}}{I_{\mathrm{m}} R}
\end{array}\right], \\
& \mathbf{B}=\left[\begin{array}{ccc}
0 & 0 & 0 \\
0 & 0 & 0 \\
0 & 0 & 0 \\
\frac{1}{I_{\mathrm{h}}} & 0 & 0 \\
0 & \frac{1}{2 r_{\mathrm{p}} M_{\mathrm{r}}}-\frac{1}{2 I_{\mathrm{e}}} & 0 \\
0 & 0 & \frac{k_{\mathrm{a}}}{I_{\mathrm{m}} R}
\end{array}\right] \\
& \mathbf{C}=\left[\begin{array}{cccccc}
0 & -N^{2} K_{\mathrm{m}} & N K_{\mathrm{m}} & 0 & 0 & 0 \\
K_{\mathrm{s}} & -K_{\mathrm{s}} & 0 & 0 & 0 & 0
\end{array}\right] \text {, } \\
& \mathbf{D}=\left[\begin{array}{lll}
0 & 0 & 0 \\
0 & 0 & 0
\end{array}\right] \text {, }
\end{aligned}
$$

here, $M_{\mathrm{R}}=\left(M_{\mathrm{r}}+I_{\mathrm{e}} / r_{\mathrm{p}}^{2}\right)$ and $C_{\mathrm{R}}=\left(\left(C_{\mathrm{r}}+C_{\mathrm{e}}\right) / r_{\mathrm{p}}^{2}\right)$. 


\section{Nomenclature}

$\alpha$ : $\quad$ Steering wheel angle

$\delta$ : $\quad$ Steering pinion angle

$\theta: \quad$ Motor angle

$C_{\mathrm{e}}$ : Output shaft viscous damping

$C_{\mathrm{m}}$ : Motor viscous damping

$C_{\mathrm{r}}$ : Viscous damping of the pinion-rack

$C_{\mathrm{s}}$ : Steering column viscous damping

$F_{\mathrm{TR}}$ : Steering resistance force

$I_{\mathrm{e}}$ : Output shaft moment of inertia

$I_{\mathrm{m}}$ : Motor moment of inertia

$I_{\mathrm{s}}$ : Steering column moment of inertia

$K_{\mathrm{a}}$ : Electromagnetic torque coefficient

$K_{\mathrm{b}}$ : Back electromotive force constant

$K_{\mathrm{m}}$ : Motor rigidity coefficient

$K_{\mathrm{r}}$ : Tie rod equivalent spring coefficient

$K_{s}$ : $\quad$ Steering column stiffness

$M_{\mathrm{r}}$ : Mass of the pinion-rack

$N$ : $\quad$ Motor gear ratio

$R: \quad$ Motor resistance

$r_{\mathrm{p}}$ : Pinion radius

$T_{\mathrm{a}}$ : $\quad$ Motor assist torque

$T_{\mathrm{c}}$ : $\quad$ Steering torque

$T_{\mathrm{h}}$ : Driver torque

$T_{\mathrm{m}}$ : Motor electromagnetic torque

$T_{\mathrm{w}}$ : Output shaft reaction torque

$u$ : Motor voltage

$x_{\mathrm{r}}$ : $\quad$ Steering rack displacement.

\section{Data Availability}

The data used to support the findings of this study are available from the corresponding author upon request.

\section{Conflicts of Interest}

The authors of this paper declare that there are no conflicts of interest regarding the publication of this paper.

\section{Acknowledgments}

This project was supported by Natural Science Foundation of China (Grant no. 51675423) and also was supported by the Key Laboratory of Road Construction Technology and Equipment (Chang'an University), MOE (Grant no. 310825171106).

\section{References}

[1] M. Parmar and J. Y. Hung, "A sensorless optimal control system for an automotive electric power assist steering system," IEEE Transactions on Industrial Electronics, vol. 51, no. 2, pp. 290-298, 2004.

[2] B. Ma, Y. Liu, Y. Gao et al., "Estimation of vehicle sideslip angle based on steering torque," International Journal of Advanced Manufacturing Technology, vol. 94, no. 9-12, pp. 3229-3237, 2016.

[3] C. Li, H. Li, Y. Chen et al., "Model-based sensor fault detection and isolation method for a vehicle dynamics control system," Proceedings of the Institution of Mechanical Engineers, Part D:
Journal of Automobile Engineering, vol. 231, no. 2, pp. 147160, 2016.

[4] M. Andersson, "Fault diagnosis of a fixed wing UAV using hardware and analytical redundancy," Electrical Engineering, Electronic Engineering, Information Engineering, Institution for Vehicular Systems, 2013.

[5] R. Isermann, R. Schwarz, and S. Stolzl, "Fault-tolerant driveby-wire systems," IEEE Transactions on Control Systems Technology, vol. 22, no. 5, pp. 64-81, 2002.

[6] R. Ghimire, C. Sankavaram, A. Ghahari et al., "Integrated model-based and data-driven fault detection and diagnosis approach for an automotive electric power steering system," in Proceedings of IEEE Autotestcon, vol. 124, pp. 70-77, Baltimore, MD, USA, September 2011.

[7] M. Lawson and X. Chen, "Hardware-in-the-loop simulation of fault tolerant control for an electric power steering system," in Proceedings of IEEE Vehicle Power \& Propulsion Conference, Harbin, China, September 2008.

[8] S. Cholakkal and X. Chen, "Fault tolerant control of electric power steering using h-infinity filter-simulation study," in Proceedings of 35th Annual Conference of IEEE Industrial Electronics Society, Porto, Portugal, November 2009.

[9] R. Wang, H. Jing, H. R. Karimi et al., "Robust fault-tolerant $H$ control of active suspension systems with finite-frequency constraint," Mechanical Systems and Signal Processing, vol. 62-63, no. 4702, pp. 341-355, 2015.

[10] H. Tohidi, K. Erenturk, and S. Shoja-Majidabad, "Passive fault tolerant control of induction motors using nonlinear block control," Control Engineering \& Applied Informatics, vol. 19, no. 1, pp. 49-58, 2017.

[11] M. Zhang, X. Liu, and F. Wang, "Backstepping based adaptive region tracking fault tolerant control for autonomous underwater vehicles," Journal of Navigation, vol. 70, no. 1, pp. 184-204, 2016.

[12] D. C. Wang, C. Long, and C. Huang, "Research on sensor fault- tolerant control based on Riccati equation for electric power steering," Key Engineering Materials, vol. 464, pp. 86-89, 2011.

[13] C. Huang, L. Chen, K. Zhang et al., "Sensor fault-tolerant control of electric power steering for electric vehicles," in Proceedings of the Fifth International Conference on Transportation Engineering, Dalian, China, September 2015.

[14] J. da Fonseca Neto, I. S. Abreu, and F. N. Da Silva, "Neural-genetic synthesis for state-space controllers based on linear quadratic regulator design for eigenstructure assignment," IEEE Transactions on Systems, Man, and Cybernetics, Part B (Cybernetics), vol. 40, no. 2, pp. 266-285, 2010.

[15] H. Asadi, S. Mohamed, C. P. Lim et al., "Robust optimal motion cueing algorithm based on the linear quadratic regulator method and a genetic algorithm," IEEE Transactions on Systems, Man, and Cybernetics-Part A: Systems and Humans, vol. 99, pp. 1-17, 2016.

[16] S. L. Schulz, H. M. Gomes, and A. M. Awruch, "Optimal discrete piezoelectric patch allocation on composite structures for vibration control based on GA and modal LQR," Computers \& Structures, vol. 128, pp. 101-115, 2013.

[17] V. K. Elumalai and R. G. Subramanian, "A new algebraic LQR weight selection algorithm for tracking control of $2 \mathrm{DoF}$ torsion system," Archives of Electrical Engineering, vol. 66, no. 1, 2017.

[18] Y. G. Liao and H. I. Du, "Modeling and analysis of electric power steering system and its effect on vehicle dynamic behavior," International Journal of Vehicle Autonomous Systems, vol. 1, no. 2, pp. 153-166, 2003. 
[19] T. Zhou, Z. Liu, L. I. Mengqi et al., Electronic Power Steering System and Its Key Technologies, Machine Tool \& Hydraulics, vol. 40, no. 7, pp. 176-179, 2012.

[20] A. Marouf, M. Djemai, C. Sentouh et al., "A new control strategy of an electric-power-assisted steering system," IEEE Transactions on Vehicular Technology, vol. 61, no. 8, pp. 3574-3589, 2012.

[21] R. C. Chabaan, "Torque estimation in electrical power steering systems," in Proceedings of IEEE Vehicle Power and Propulsion Conference, Dearborn, MI, USA, September 2009.

[22] C. Chen and A. Holohan, "Stability robustness of linear quadratic regulators," International Journal of Robust and Nonlinear Control, vol. 26, no. 9, pp. 1817-1824, 2015.

[23] C. Scherer and S. Weiland, Linear Matrix Inequalities in Control, Lecture Notes, Dutch Institute for Systems and Control, Netherlands, 2000.

[24] V. Kumare and J. Jerome, "Algebraic Riccati equation based Q and R matrices selection algorithm for optimal LQR applied to tracking control of 3rd order magnetic levitation system," Archives of Electrical Engineering, vol. 65, no. 1, pp. 151-169, 2016.

[25] F. Borrelli and T. Keviczky, "Distributed LQR design for identical dynamically decoupled systems," IEEE Transactions on Automatic Control, vol. 53, no. 8, pp. 1901-1912, 2008.

[26] J. J. Grefenstette, "Optimization of control parameters for genetic algorithms," IEEE Transactions on Systems, Man, and Cybernetics, vol. 16, no. 1, pp. 122-128, 1986.

[27] D. Zhou and Y. Ye, Modern Fault Diagnosis and Fault Tolerant Control, Tsinghua University Press, Beijing, China, 2000 .

[28] G. Xie and G. Meng, "Fault-tolerant control strategy with integrity and robustness for an electric power-steering system," Mechanical Science and Technology for Aerospace Engineering, vol. 30, no. 3, pp. 429-434, 2011. 


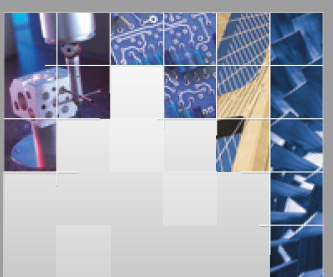

\section{Enfincering}
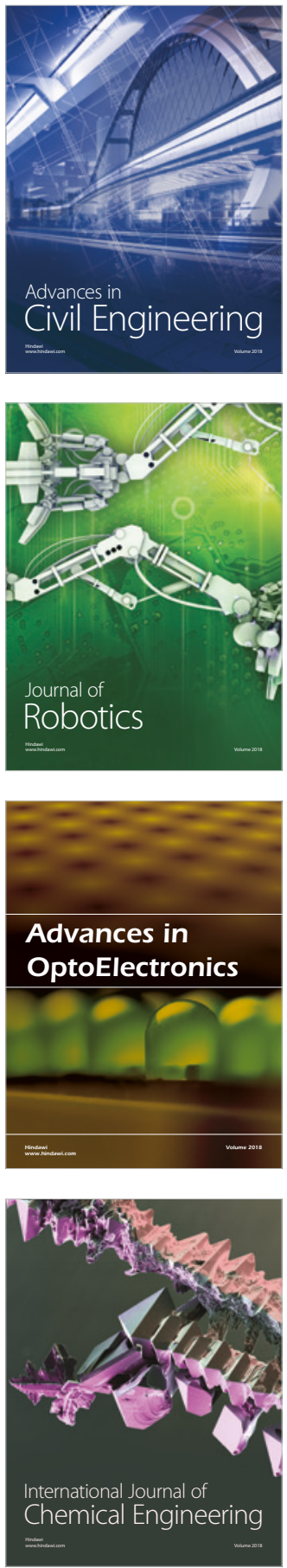

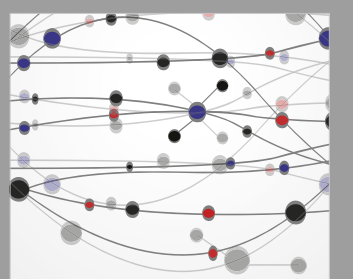

\section{Rotating \\ Machinery}

The Scientific World Journal

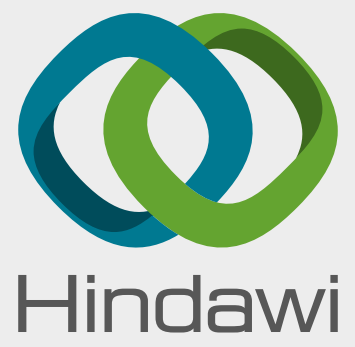

Submit your manuscripts at

www.hindawi.com
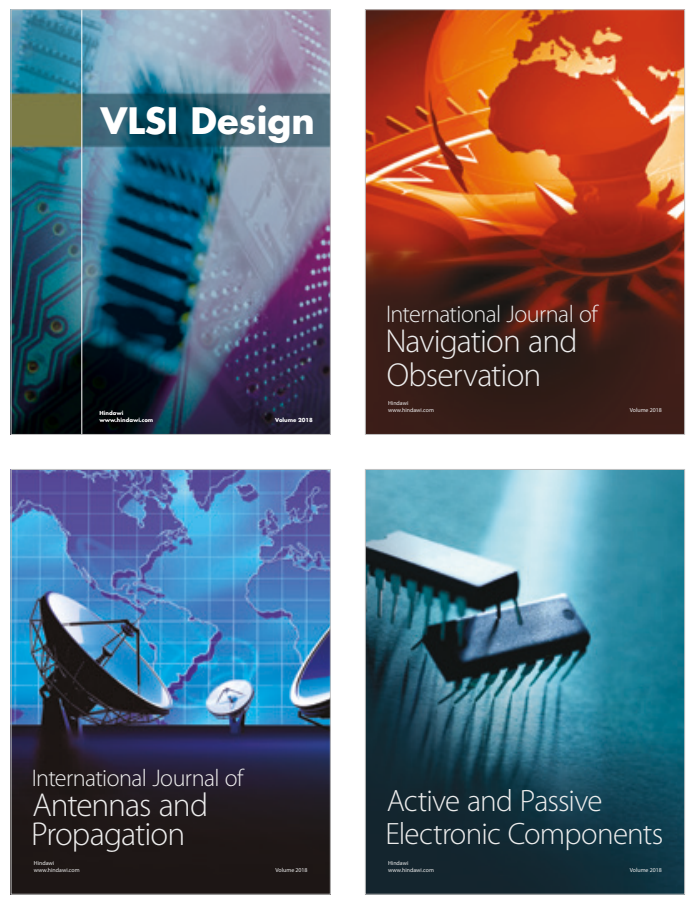
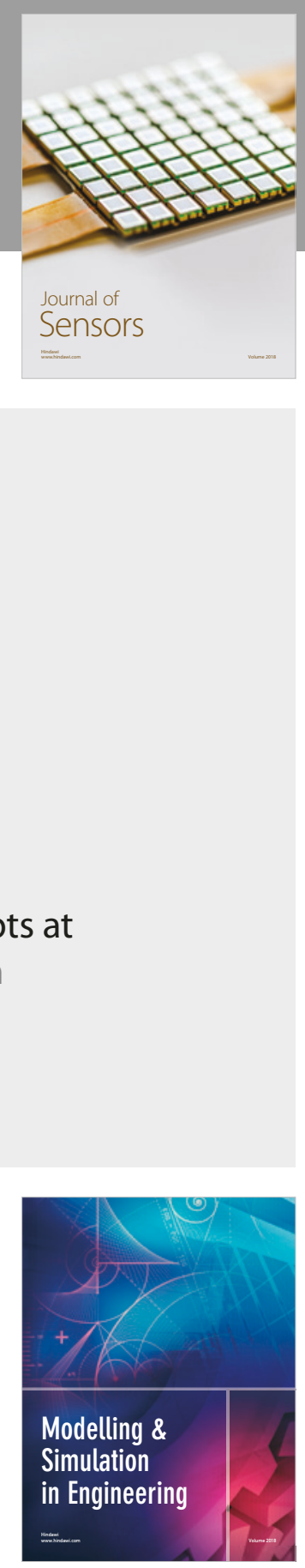

\section{Advances \\ Multimedia}
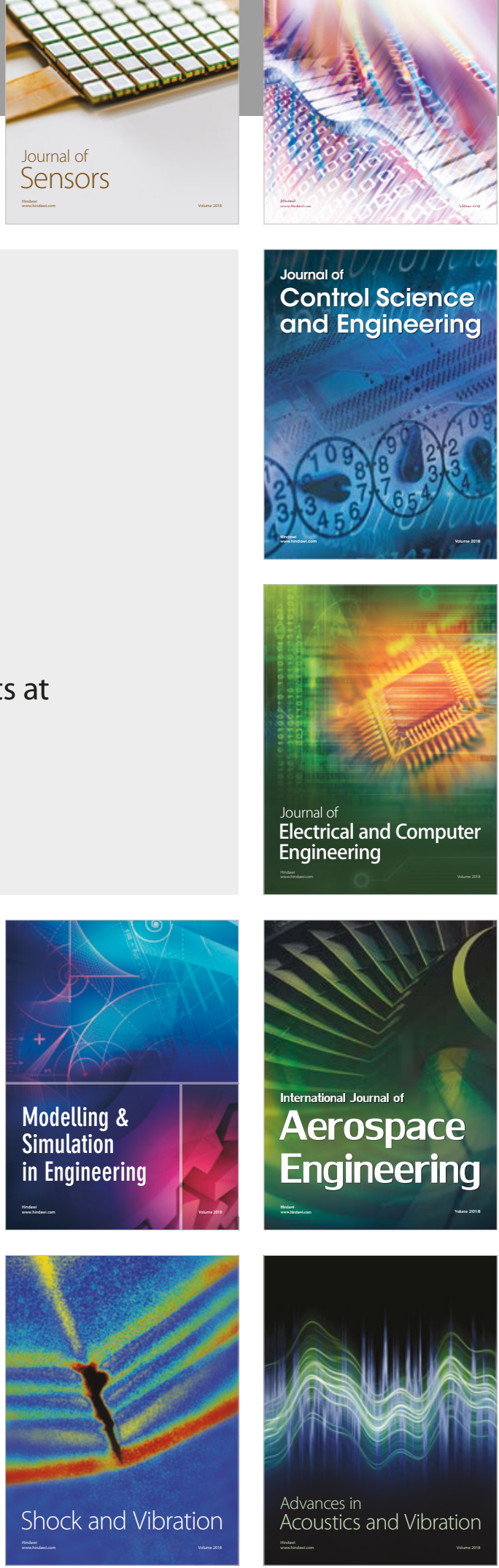